\title{
Effects of internal molecular degrees of freedom on the thermal conductivity of some glasses and disordered crystals
}

\author{
A. I. Krivchikov, ${ }^{1}$ O. A. Korolyuk, ${ }^{1}$ I. V. Sharapova, ${ }^{1}$ J. Ll. Tamarit,,${ }^{2,}$ F. J. Bermejo, ${ }^{3}$ L. C. Pardo, ${ }^{2}$ M. Rovira-Esteva, ${ }^{2}$ \\ M. D. Ruiz-Martin, ${ }^{2}$ A. Jezowski, ${ }^{4}$ J. Baran, ${ }^{4}$ and N. A. Davydova ${ }^{5}$ \\ ${ }^{1}$ B. Verkin Institute for Low Temperature Physics and Engineering of NAS Ukraine, 47 Lenin Avenue, Kharkov 61103, Ukraine \\ ${ }^{2}$ Grup de Caracterització de Materials, Departament de Física i Enginyieria Nuclear, ETSEIB, Universitat Politècnica de Catalunya, \\ Diagonal 647, E-08028 Barcelona, Catalonia, Spain \\ ${ }^{3}$ Instituto de Estructura de la Materia, CSIC, Serrano 123, E-28006 Madrid and Department of Electricity and Electronics, \\ University of the Basque Country, P.O. Box 664, E-48080 Bilbao, Spain \\ ${ }^{4}$ Institute for Low Temperature and Structure Research, Polish Academy of Sciences, PN 1410, PL-50-950 Wroclaw, Poland \\ ${ }^{5}$ Institute of Physics, NANU 46 pr. Nauki, 03028 Kiev, Ukraine
}

(Received 19 October 2011; revised manuscript received 12 January 2012; published 30 January 2012)

\begin{abstract}
The thermal conductivity $\kappa(T)$ of the fully ordered stable phase II, the metastable phase III, the orientationally disordered (plastic) phase I, as well as the nonergodic orientational glass (OG) phase, of the glass former cyclohexanol $\left(\mathrm{C}_{6} \mathrm{H}_{11} \mathrm{OH}\right)$ has been measured under equilibrium vapor pressure within the $2-200 \mathrm{~K}$ temperature range. The main emphasis is here focused on the influence of the conformational disorder upon the thermal properties of this material. Comparison of results with those regarding cyanoclyclohexane $\left(\mathrm{C}_{6} \mathrm{H}_{11} \mathrm{CN}\right)$, a chemically related compound, serves to quantify the role played by the terminal groups $-\mathrm{OH}$ and $-\mathrm{CN}$ on the phonon scattering processes. The picture that emerges shows that motions of such groups do play a minor role as scattering centers, both within the low-temperature orientationally ordered phases as well as in the OG states. The results are analyzed within the Debye-Peierls relaxation time model for isotropic solids comprising mechanisms for long-wave phonon scattering within the OG and orientational ordered low-temperature phases, as well as others arising from localized short-wavelength vibrational modes as pictured by the Cahill-Pohl model. By means of complementary neutron and Raman scattering we show that in the OG state the energy landscapes for both compounds are very similar.
\end{abstract}

DOI: 10.1103/PhysRevB.85.014206

PACS number(s): 66.70.-f

\section{INTRODUCTION}

The low-temperature properties of disordered solids exhibit striking differences with respect to those shown by their fully ordered crystalline counterparts. These manifest themselves as excess, low-energy excitations. The nature of these excitations as well as their role to explain the macroscopic properties of glasses are still open questions. ${ }^{1-4}$ To unravel the underlying physics, studies on relatively simple molecular systems which can be tailored to show controlled amounts of disorder pertaining to the molecular centers of mass, the relative molecular orientations, or even the molecular conformations that arise from flexible molecular segments showing different orientations with respect to some molecule-fixed frame constitute important benchmarks. ${ }^{5-7}$ Orientational glasses (OGs) are materials where, in contrast to fully amorphous materials such as the structural glasses (SGs), the centers of mass of the molecules sit on the nodes of a crystalline lattice, keeping the molecular orientations and/or molecular conformations randomized. The beauty of OGs stems from their remarkably close thermal and dynamic properties to those exhibited by amorphous materials. $^{8-11}$

Some attempts have been registered to account for the excess in the density of low-energy states in glasses. Such states generate the boson peak, which results from the excess in the vibrational density of states, $g(\omega)$, over the classical Debye $\left[g_{D}(\omega) \propto \omega^{2}\right]$ value and gives rise to well-known anomalies in the heat capacity and thermal conductivity. ${ }^{12,13}$ In order to treat departures from the classical Debye model several approaches have been proposed and might be classified into two groups.
A first type considers the existence of a harmonic elastic disorder medium with fluctuating force constants distributed according to some idealized distribution. ${ }^{14-17}$ Alternatively, other approaches assume the existence of quasilocal lowenergy vibrations resulting from librations of structural units able to scatter sound waves. Such phenomenological models have been able to account for the anomalies in the heat capacity as well as the thermal conductivity. Such models consist in generalizations of the tunneling model, such as the soft potential model (SPM). ${ }^{18-21}$

The thermal conductivity for disordered materials has been rationalized in terms of the contribution of propagating phonons and other diffusive modes. ${ }^{5-7,22}$ For materials composed by molecules with low-lying internal degrees of freedom such as 1,2-difluoro-1,1,2,2-tetrachloroethane $\left(\mathrm{CFCl}_{2}-\mathrm{CFCl}_{2}\right.$, freon 112) its thermal properties have been rationalized as resulting from the action of two nonequivalent molecular conformations, trans and gauche, which have been claimed as the origin of a higher density of minima in the potentialenergy landscape. ${ }^{10}$ Similar results concerning such molecular isomeric effects were found for the structural glass obtained from 1-propanol. ${ }^{5,7}$ Thus, although the soft-potential model was able to describe the low-temperature values of thermal conductivity, a term accounting for resonant scattering of phonons by simple oscillators was required to account for the wide temperature plateau appearing in molecular compounds with conformational disorder. $5,7,10$

The rationale behind the action of these additional molecular degrees of freedom on macroscopic thermal properties of 
the materials in question portrays them as able to hybridize with the sound modes, and thus the origin of the resonant scattering contribution to the thermal conductivity is explained as the consequence of a coupling between the resonant scattering from quasilocal vibrations and the low-energy molecular librational modes. ${ }^{18,23,24}$

Materials composed by ring molecules such as as cyclohexanol $\left(\mathrm{C}_{6} \mathrm{H}_{11} \mathrm{OH}\right.$, hereinafter denoted by $\left.\mathrm{C}_{6} \mathrm{OH}\right)$ and cyanocyclohexane $\left(\mathrm{C}_{6} \mathrm{H}_{11} \mathrm{CN}, \mathrm{C}_{6} \mathrm{CN}\right)$ are interesting probes to unravel the effects of different kinds of disorder on thermal properties. In addition to the shining evidence of the effects of whole-body molecular rotations and chemical isomerism on structure and thermal properties, $\mathrm{C}_{6} \mathrm{OH}$ and $\mathrm{C}_{6} \mathrm{CN}$ enable us to quantify the role of conformational changes upon a fundamental property such as thermal conductivity. The phase behavior of the archetypical glass former $\mathrm{C}_{6} \mathrm{OH}$ was early disclosed by calorimetry and other means. ${ }^{25-32}$ The liquid freezes at $299 \mathrm{~K}$ into an orientationally disordered (OD) (plastic or rotator phase, phase I) face-centered-cubic phase, ${ }^{28-30}$ where molecules rotate nearly freely about the crystal lattice nodes. Further cooling down to $265.5 \mathrm{~K}$ leads to a transformation into the ordered stable phase II, recently characterized as tetragonal structure ( $P \overline{4} 2_{1} c$ space group) ${ }^{30}$ The stable phase I easily undercools under moderate cooling rates and shows a glass transition at $148-150 \mathrm{~K}$, giving rise to an OG. ${ }^{25-27,31,32}$ By heating up the OG, and after crossing the glass transition, a metastable ordered monoclinic ( $P c$ space group) phase III appears at $\sim 200 \mathrm{~K}$. This phase remains metastable if cooled down. Alternatively, it enters phase II after heating up to $220-240 \mathrm{~K}$. An additional metastable form

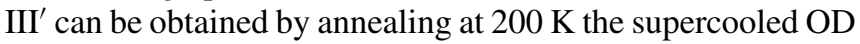
phase I.

The intriguing polymorphic behavior of $\mathrm{C}_{6} \mathrm{OH}$ has been long attributed to the pseudospherical-shaped molecule combined with the possibility to display several conformations. The three-dimensional structure of the ring of this cyclic molecule can display several puckered out-of-plane conformations and, in addition, the polar-OH group may adopt either of two conformations, the axial or the equatorial, with respect to the carbon atom on which the group is bonded. ${ }^{33}$ For each of these conformations the hydroxyl group can be aligned itself in- or out-of-plane. Despite the general belief that polymorphic forms concern the different possible conformations of the molecule and the large number of experimental reports on $\mathrm{C}_{6} \mathrm{OH}$, it has been recently found that for all the ordered phases $\mathrm{C}_{6} \mathrm{OH}$ adopts the chair conformation for the ring whereas the $-\mathrm{OH}$ group lies in the equatorial orientation (both inand out-of-plane orientations). ${ }^{30}$ This is not the case for the OD phase I, for which the presence of all conformations has been reported $^{28,29}$ with small energetic barriers between the axial and equatorial isomers. For $\mathrm{C}_{6} \mathrm{OH}$ and similar cyclic compounds it has been shown that disorder in the glassy crystal phase arises from some degree of disorder in the whole molecular orientation and from a mixture of conformations due to the difficulties of the system in finding the regular packing in a nonequilibrium state. ${ }^{25}$

Also, $\mathrm{C}_{6} \mathrm{CN}$ shows rich polymorphism. The stable liquid crystallizes at $285 \mathrm{~K}$ into an OD phase I, the symmetry of which is also face-centered cubic. On further cooling a glass transition appears at approximately $135 \mathrm{~K}$ as proven by calorimetry and dielectric spectroscopy. ${ }^{8,34-38}$ An additional, small heat-capacity jump ${ }^{39}$ also appears at around $55 \mathrm{~K}$, which has been attributed to the freezing of the interconversion between the axial and equatorial conformers. ${ }^{40}$

A stable low-temperature phase II results by annealing of phase I. Heating this phase leads to a transformation into the OD phase I at $217 \mathrm{~K}$. Notice however that below this temperature phase I remains stable enough to be studied on considerably long time scales. No structural studies have been reported for phase II, so the assumption that it is an orientationally ordered phase of $\mathrm{C}_{6} \mathrm{CN}$ is only based upon the enthalpy changes reported for the II to I phase transition. ${ }^{40}$ Preliminary x-ray diffraction patterns seem to indicate a strong decrease of lattice symmetry with respect to the OD phase I.

As aforementioned for $\mathrm{C}_{6} \mathrm{OH}, \mathrm{C}_{6} \mathrm{CN}$ is a flexible molecule and thus several conformers can be also observed. In addition to the ring conformations, depending on the position of the carbonitrile group with respect to the cyclohexane ring, axial and equatorial conformations exist. Interconversion between those involves an energy barrier of $\sim \Delta E / k_{B}=4500 \mathrm{~K}$ according to infrared and Raman measurements. ${ }^{41-43}$ Within the liquid and OD I states both conformations are equally populated, whereas for the low-temperature phase II only the axial chair conformation exists. ${ }^{41-43}$

The present work reports on phonon scattering phenomena taking place within the ordered and disordered solid phases of $\mathrm{C}_{6} \mathrm{OH}$ and $\mathrm{C}_{6} \mathrm{CN}$ and their experimentally available nonergodic states from measurements of thermal conductivities. The results are analyzed within the well-known Debye-Peierls relaxation time model for isotropic solids assuming different mechanisms of long-wave phonon scattering within the OG and orientational ordered phases, and an additional mechanism described by localized short-wavelength vibrational modes according to the Cahill-Pohl model. Details of the experimental procedures are given in Sec. II. Results, presented in Sec. III, are divided in two parts, corresponding to the orientationally disordered and ordered states. Sec. IV is devoted to the discussion of the results under the framework of several theoretical models. Finally, conclusions are sketched in the last section, Sec. V.

\section{EXPERIMENTAL}

\section{A. Materials}

Cyclohexanol and cyanocyclohexane were purchased from Aldrich with purities of $99 \%$ and used without further purification after temperature and enthalpy of melting were checked by means of scanning calorimetry to be within the standard values reported in the literature. ${ }^{25,40}$ Both compounds were always handled with caution under Ar-controlled atmosphere.

\section{B. Thermal conductivity measurements}

The thermal conductivity of the solid samples was measured under equilibrium vapor pressure using two experimental setups based upon the steady-state method. The OG state was reached by fast cooling (above $50 \mathrm{~K} \mathrm{~min}^{-1}$ ) from the OD phases through the glass transition region to the boiling temperature of liquid $\mathrm{N}_{2}$. The thermal conductivity of the OG state of both $\mathrm{C}_{6} \mathrm{OH}$ and $\mathrm{C}_{6} \mathrm{CN}$ was measured at 
gradually decreasing temperature from 77 to $2 \mathrm{~K}$ and then increasing temperature up to $160 \mathrm{~K}$ with an experimental setup detailed elsewhere. ${ }^{44}$ The experience gained in the detailed measurements on a variety of cryocrystals by the groups at Kharkov and Wroclaw shows that random measurement errors can be as low as $2 \%$. In turn, systematic errors arising from measurement of geometrical parameters such as the inner container cross sections or the spacing between thermocouples amount to an additional systematic error that can reach $10 \%$ of the measured property (for a review see compendium of Ref. 45).

The low-temperature orientationally ordered phases of $\mathrm{C}_{6} \mathrm{OH}$ were reached by procedures described by Adachi et al. ${ }^{25}$ The ordered phase of $\mathrm{C}_{6} \mathrm{CN}$ was achieved by heating the supercooled phase I up to $205 \mathrm{~K}$ and further annealing for 50 h. $^{39}$

Thermal conductivity measurements for the ordered phase $\mathrm{C}_{6} \mathrm{CN}$ were carried out by means of the axial stationary heat flow method in the 4-206 $\mathrm{K}$ range on heating and cooling. Polypropylene ampoule sample containers $(9 \mathrm{~mm}$ inner diameter, $18 \mathrm{~mm}$ long, and $0.9 \mathrm{~mm}$ wall thickness) were kept cold by anchoring them into a thick copper panel suspended within a cryostat. Temperature of the surroundings was measured with germanium and platinum thermometers and stabilized within $\pm 3 \mathrm{mK}$. Sample temperatures were recorded by constantan-manganin thermocouples using liquid nitrogen and liquid helium as reference points. The temperature difference along the sample (typically around $0.2-0.3 \mathrm{~K}$ ) was generated by an electrical heater. In order to avoid heat losses, high vacuum and four shields were used.

\section{Neutron scattering measurements}

The density of states of the OG phases was measured by inelastic neutron scattering spectroscopy. The experiments were performed using the TOFTOF high-resolution direct geometry chopper spectrometer, located at FRM II (Garching, Germany). This instrument offers a large flexibility for wavelength selection and chopper speed which permits considerable optimization of the energy range, energy resolution, momentum transfer, and count rate. The instrument resolution was set to $55 \mu \mathrm{eV}$ (full width at half maximum) at the elastic peak position as measured using a vanadium cylinder having the same dimensions as the sample holder. This energy resolution was achieved using an incident neutron wavelength of $6 \AA$ and a chopper rotation frequency of $12000 \mathrm{rpm}$.

The data here reported correspond to the frequency distribution obtained at $50 \mathrm{~K}$. The sample was introduced inside an aluminum hollow cylinder (sample thickness of $\sim 0.1$ $\mathrm{mm}$, container wall thickness of $\sim 0.1 \mathrm{~mm}$ ) and quenched from room temperature to $50 \mathrm{~K}$, thus ensuring the formation of $\mathrm{OG}$ from phase I. The measured time of flight spectra were normalized to the elastic scattering of the vanadium cylinder previously used to obtain the instrument energy resolution. Then the detector efficiency correction was carried out. In order to subtract the background signal, the empty container was measured inside the cryostat under the same experimental conditions (instrument setup, temperature). After performing the background subtraction, the measured $S(\theta, t)$ were transformed into $S(Q, \omega)$ with the program IDA. ${ }^{46}$ The density of states was also calculated using the IDA software, taking as a starting point $S(Q, \omega)$. All the steps followed are described in Ref. 47.

\section{Raman measurements}

Depolarized low-frequency Raman spectra of $\mathrm{C}_{6} \mathrm{OH}$ samples were measured in a $90^{\circ}$ scattering configuration with a double monochromator (Jobin-Yvon Ramanor U 1000 spectrometer), equipped with a standard photomultiplier detector controlled by the Spectra Max software. The stray light rejection was $10-14$ at $20 \mathrm{~cm}^{-1}$ from the Rayleigh line. The spectral slit width was $2 \mathrm{~cm}^{-1}$. The spectra were recorded using the $514.5 \mathrm{~nm}$ line of an $\mathrm{Ar}^{+}$laser and the laser beam power focused on a sample was about $150 \mathrm{~mW}$. For measurements at low temperatures the sample was confined in thin glass capillaries which were mounted on the copper finger of a Displex closed-cycle cryostat that can be operated in the temperature range $12-330 \mathrm{~K}$.

\section{RESULTS}

\section{A. Orientational glass and orientationally disordered phase}

The thermal conductivity of the $\mathrm{OG}$ phases of $\mathrm{C}_{6} \mathrm{OH}$ and $\mathrm{C}_{6} \mathrm{CN}$ is shown in Fig. 1. Here, $\kappa(T)$ displays the typical temperature dependence for molecular orientational glasses. ${ }^{10}$ It shows a strong increase with increasing temperatures in the low-temperature domain $(2-5 \mathrm{~K})$ followed by a plateau which extends over a relatively narrow temperature range. It can be seen that $\kappa(T)$ for these OGs is, astonishingly enough, virtually the same for the whole temperature range until the glass transition region is approached, a fact that is rationalized as a proof that scattering of heat-carrying phonons from lowand medium-energy excitations should be quite similar within these disordered phases. Our data for $\mathrm{C}_{6} \mathrm{OH}$ under equilibrium vapor pressure are $\sim 10 \%$ lower than those published by Andersson et al. ${ }^{32}$ for the same range of temperatures (higher that $100 \mathrm{~K}$ ) and are about $20 \%$ and $10 \%$ higher than those from Bonjour et al. ${ }^{31}$ within the $2-20 \mathrm{~K}$ range.

The Fig. 2 shows the neutron-weighted generalized frequency distribution or density of states $g(\omega)$ obtained from neutron spectroscopy measurements. From such distributions

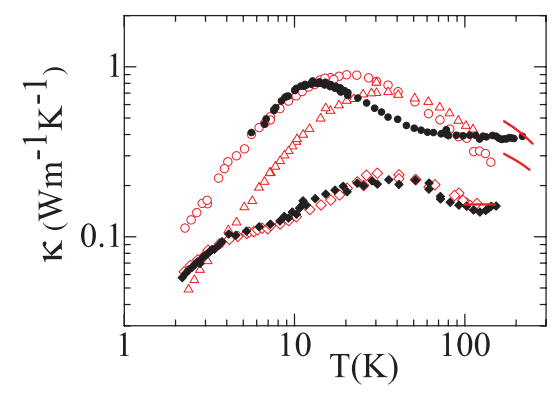

FIG. 1. (Color online) Log-log plot of the thermal conductivity as a function of temperature for crystalline phases II and III (circles and triangles, respectively) and OG (diamonds) of cyclohexanol (empty red symbols) and for crystalline phase II (full black circles) and OD and OG (full black diamonds) of cyanocyclohexane. Solid red lines correspond to data from Ref. 32 for phases II and III of cyclohexanol at $0.06 \mathrm{GPa}$. 


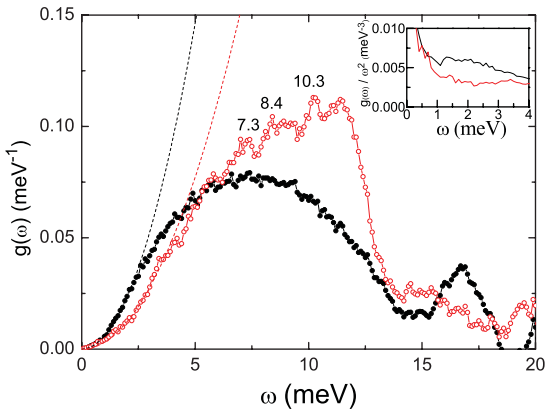

FIG. 2. (Color online) Density of states as a function of energy for $\mathrm{C}_{6} \mathrm{OH}$ (empty red circles) and $\mathrm{C}_{6} \mathrm{CN}$ (filled black circles) OGs at $50 \mathrm{~K}$ as derived from inelastic neutron scattering measurements. Dotted-dashed lines indicate the effective Debye density of states $\left[g(\omega)=3 \omega^{2} / \omega_{D}^{3}\right]$ for $\omega_{D}=9.9\left(\mathrm{C}_{6} \mathrm{OH}\right)$ and $8 \mathrm{meV}\left(\mathrm{C}_{6} \mathrm{CN}\right)$. The inset shows the density of states scaled by $\omega^{2}$ to better show the departure from the Debye behavior in the low-energy domain.

several comments are in order: First, one notices strikingly strong differences between both density distributions from energies $\sim 7 \mathrm{meV}$ onward. Second, as shown in the inset the existence of an extended peak in the low-energy region is indicative of the action of a large number of excess modes [associated with the boson peak, i.e., excess vibrational modes above the Debye prediction $g(\omega) \propto \omega^{2}$ for crystalline ordered solids], and third, both distributions remain significantly different down to very low frequencies.

The low-frequency Raman spectra of $\mathrm{C}_{6} \mathrm{OH}$ obtained on cooling from the liquid state down to the glassy state at $100 \mathrm{~K}$ are shown in Fig. 3. The spectrum of the liquid $\mathrm{C}_{6} \mathrm{OH}$ is characterized by the strong scattering structureless line (a wing of the Rayleigh line) reaching its maximum at zero-shift frequency $^{47}$ (curve a). Upon cooling, a transition to the OD phase I occurs at $296 \mathrm{~K}$. The curve b in Fig. 3 shows the Raman spectra of the OD phase $\mathrm{I}$ of $\mathrm{C}_{6} \mathrm{OH}$ recorded at $220 \mathrm{~K}$. In this spectrum the lattice vibrations appear to be slightly marked. Below the glass transition temperature at $T=100 \mathrm{~K}$ (curve c) the decrease of the Rayleigh line leads to the observation of a weak shoulder at $15.3 \mathrm{~cm}^{-1}(1.9 \mathrm{meV})$, which can be tentatively attributed to the boson peak.

\section{B. Orientationally ordered phases}

The thermal conductivity as a function of temperature for $\mathrm{C}_{6} \mathrm{OH}$ and $\mathrm{C}_{6} \mathrm{CN}$ for the stable and metastable orientationally ordered phases is shown in Fig. 1 in a double-logarithmic plot. Data for $\mathrm{C}_{6} \mathrm{OH}$ compare well with those previously published

TABLE I. Values for the $A$ and $C$ fitted parameters [Eq. (1)] for the orientationally ordered and orientationally disordered phases of cyclohexanol $\left(\mathrm{C}_{6} \mathrm{OH}\right)$ and cyanocyclohexane $\left(\mathrm{C}_{6} \mathrm{CN}\right)$.

\begin{tabular}{lccc}
\hline \hline Chemical & Phase & $A\left(\mathrm{~W} \mathrm{~m}^{-1}\right)$ & $C\left(\mathrm{~W} \mathrm{~m}^{-1} \mathrm{~K}^{-1}\right)$ \\
\hline $\mathrm{C}_{6} \mathrm{OH}$ & OG & 7.3 & 0.09 \\
& II & 26.9 & 0.10 \\
& III & 26.9 & 0.19 \\
$\mathrm{C}_{6} \mathrm{CN}$ & OG & 5.3 & 0.09 \\
& II & 3.5 & 0.36 \\
\hline \hline
\end{tabular}

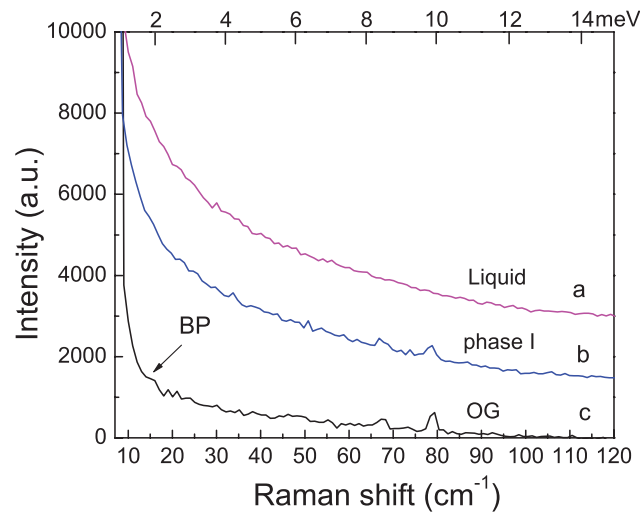

FIG. 3. (Color online) Low-frequency Raman spectra of $\mathrm{C}_{6} \mathrm{OH}$ measured on cooling from 306 down to $100 \mathrm{~K}$ (in $\mathrm{cm}^{-1}$, bottom axis, and meV, top axis): (a) liquid phase at $306 \mathrm{~K}$; (b) OD phase I at $293 \mathrm{~K}$; (c) OG state at $100 \mathrm{~K}$. BP is attributed to the boson peak at $15.3 \mathrm{~cm}^{-1}$ $(1.9 \mathrm{meV})$ on the spectrum of OG.

by Andersson et al. ${ }^{32}$ at high pressure. Thermal conductivity for both phases displays typical temperature dependence for ordered phases. Nevertheless, it should be emphasized that $\kappa(T)$ for the metastable phase III is higher than that for the stable phase II at temperatures higher than $50 \mathrm{~K}$, a unique case as first stressed by Andersson et al. ${ }^{32}$

As for the orientationally ordered phases $\kappa(T)$ increases with temperature as $T^{3 / 2}$ till the phonon maximum around 20 and $35 \mathrm{~K}$ for the stable phase II and the metastable phase III of $\mathrm{C}_{6} \mathrm{OH}$, whereas such a maximum is reached at $\sim 15 \mathrm{~K}$ for the ordered phase of $\mathrm{C}_{6} \mathrm{CN}$. The typical bell-like shape for $\kappa(T)$ for orientationally ordered phases in such a log-log plot is clearly observed and thus, after the maximum, $\kappa(T)$ decreases with temperature, although the decrease is slightly weaker than the expected law $T^{-1} .^{22}$ Such a maximum is known to mark the transition from phonon-phonon scattering to phonon scattering on defects in ordered crystals. ${ }^{48-50}$

To highlight such deviation from the $T^{-1}$ law, Fig. 4 shows $\kappa(T) \cdot T$ in the form

$$
\kappa(T)=\frac{A}{T}+C .
$$

The first term on the right side of Eq. (1) accounts for threephonon processes, whereas the second term $(C)$ describes the additional mechanism which causes the thermal conductivity

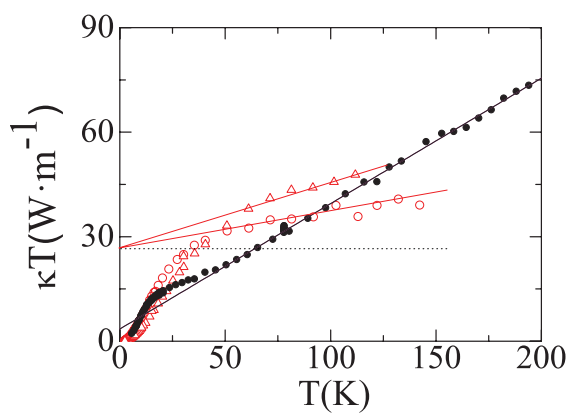

FIG. 4. (Color online) Thermal conductivity times temperature as a function of temperature for the orientationally ordered phases. Symbols as in Fig. 1. 


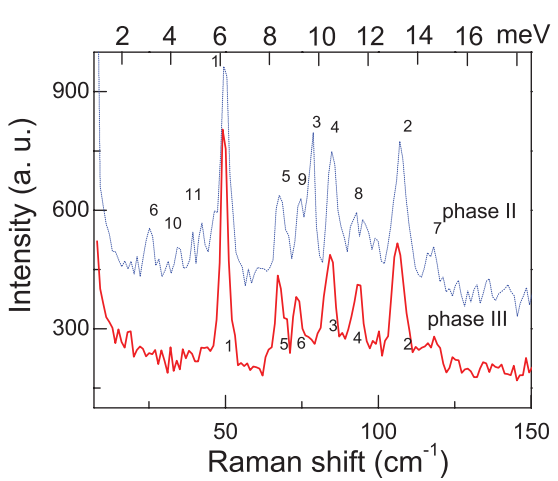

FIG. 5. (Color online) Low-frequency Raman spectra (in $\mathrm{cm}^{-1}$, bottom axis, and meV, top axis) of the stable (II) (dotted blue line) and metastable (III) (red line) phases of $\mathrm{C}_{6} \mathrm{OH}$ at $100 \mathrm{~K}$.

to deviate from the $T^{-1}$ law at temperatures beyond the phonon maximum. ${ }^{6,22}$ Figure 4 displays the temperature domain in which each of the analyzed phases deviates from the common behavior. For the stable phase II and metastable phase III of $\mathrm{C}_{6} \mathrm{OH}$, the constant term $(C)$ in Eq. (1) appears to be relevant at around $50 \mathrm{~K}$, whereas for the orientationally ordered phase II of $\mathrm{C}_{6} \mathrm{CN}$ deviation appears at $\sim 40 \mathrm{~K}$ and the second term becomes dominant at $T>70 \mathrm{~K}$. It is worth noting that the $A$ values [intersection between the straight lines and the $\kappa(T) \cdot T$ axis] are virtually the same but the $C$ values differ by a factor of about 2 for both orientationally ordered phases of $\mathrm{C}_{6} \mathrm{OH}$, as shown in Table I. On the contrary, for the ordered phase of $\mathrm{C}_{6} \mathrm{CN}$ the $A$ value is considerably smaller which means that there is strong scattering whereas the $C$ value is considerably larger. In $\mathrm{C}_{6} \mathrm{OH}$ the $C$ value for phase III is larger than that for phase II. The $C$ values for $\mathrm{C}_{6} \mathrm{OH}$ are similar to $C$ values for primary alcohols in the orientationally ordered state. ${ }^{51}$

The low-frequency Raman spectra of the stable II and metastable III phases of $\mathrm{C}_{6} \mathrm{OH}$ at $100 \mathrm{~K}$ are shown in Fig. 5 . The transformation from phase I to phase III occurs in such a way that the spectrum within the lattice region changes from two broad bands in phase I to six narrow bands. Polymorphic transformation from phase III to phase II reveals new intramolecular modes. The existence of the set of narrow lines in Raman spectra in both III and II phases thus monitors the development of translational and orientational long-range order.

It can be seen that the lattice vibration frequencies in the Raman spectra of the ordered phases are quite different, while the spectrum of the stable crystal II shows the lowest intermolecular (lattice) vibrations centered at $25.1 \mathrm{~cm}^{-1}$ which appears as a well-defined phonon peak; the spectrum of the metastable crystal III shows well-resolved Raman bands above $49.8 \mathrm{~cm}^{-1}$ only.

\section{DISCUSSION}

As is well known, the analysis of the temperature dependence of the thermal conductivity for solids other than simple crystals has to be carried out using heuristic models which stem from the pioneering work of Callaway, ${ }^{52}$ who included basic mechanisms for the scattering of heat-carrying phonons such as scattering from grain boundaries and on dislocations by stress fields, three-phonon processes, and scattering from point defects. For materials composed by molecular substances, excitations other than acoustic phonons such as low-frequency molecular rotations and low-angle librations need to be taken care of. The difficulties hampering such an endeavor are twofold. First, the spectrum of collective excitations is known with enough detail for only a few systems such as most of the diatomic solids and others composed for molecules having high point-group symmetries. Second, development of a theory which significantly accounts for all the relevant kinetic processes leading to scattering of sound waves has been shown to be a very difficult task. In fact, to the authors' knowledge, most of the efforts to develop such a theory stop with the inclusion of details of low-frequency librations in much the same way as reported by Slusarev and Freiman ${ }^{53}$ and Konstantinov. ${ }^{54}$ Under such circumstances the analysis here carried out proceeds on phenomenological grounds, following approaches which have been proven to rely on physically sound results when analyzing data for simpler systems.

In what follows we will cast the temperature dependence of the thermal conductivity in the solid phases of $\mathrm{C}_{6} \mathrm{OH}$ and $\mathrm{C}_{6} \mathrm{CN}$ as given by the sum of two contributions: ${ }^{54-59}$

$$
\kappa(T)=\kappa_{\mathrm{ph}}(T)+\kappa_{\min }(T),
$$

where $\kappa_{\mathrm{ph}}(T)$ corresponds to the propagating acoustic phonons with long relaxation times $\tau_{R}(\omega, T)$ typical for resistive phonon scattering. The second component, $\kappa_{\min }(T)$, accounts for shortwavelength acoustic phonons with the minimum allowed value of the phonon relaxation time $\tau_{\min }(\omega)=\pi / \omega$ according to the model developed by Cahill-Pohl. ${ }^{60}$

The temperature dependence of $\kappa_{\mathrm{ph}}(T)$ can be described by the relaxation time model (the Debye-Peierls model): ${ }^{48,61}$

$$
\kappa_{\mathrm{ph}}(T)=\frac{k_{B}^{4} T^{3}}{2 \pi^{2} \hbar^{3} c_{s}} \int_{0}^{\theta_{D} / T} \tau_{R}(x) \frac{x^{4} e^{x}}{\left(1-e^{x}\right)^{2}} d x,
$$

where $x=\hbar \omega / k_{B} T, \theta_{D}$ stands for the Debye temperature, $c_{s}$ is the velocity of sound averaged over longitudinal and transverse polarizations, and $\tau_{R}(x)$ is an effective relaxation time for phonon scattering which encloses the different scattering phenomena, which limit heat transfer. The model is used for the description of $\kappa_{\mathrm{ph}}(T)$ for both crystalline and amorphous solids when $\tau_{R}(\omega) \gg \tau_{\min }(\omega)$ and $\kappa_{\mathrm{ph}}(T) \propto T^{-1}$ corresponds to the case of phonon-phonon scattering at high temperature. ${ }^{55,58}$

As referred to above, the simplest description of $\kappa_{\min }(T)$ is provided by the phenomenological Cahill-Pohl model which has been used time and again to analyze the thermal conductivity of amorphous solids at high temperatures, $\kappa_{\min }(T)=$ $\kappa_{C P}(T),{ }^{60}$

$$
\kappa_{\min }(T)=\kappa_{C P}(T)=\frac{k_{B}^{3} T^{2}}{2 \pi^{2} \hbar^{3} c_{s}} \int_{0}^{\theta / T} \frac{x^{3} e^{x}}{\left(1-e^{x}\right)^{2}} d x .
$$

Within such model all acoustic phonons have a minimal mean-free path set to be equal to one half of their wavelength. $\kappa_{C P}(T)$ corresponds to the minimal thermal conductivity of a solid with very strong resistive phonon scattering, $\tau_{R}(\omega) \ll$ $\tau_{\min }(\omega)$. At high temperatures $\kappa_{C P}(T)$ is nearly temperature independent. The Debye approximation which disregards the polarization modes assumes that the minimum lifetime of each vibration is equal to its half-period time. Later on the CahillPohl model was developed by Klinger and Halpern ${ }^{62}$ who 
built up a theoretical model of soft-mode dynamics to describe vibrational terahertz anomalies for glasses.

A thermal conductivity crossover from $\kappa_{\mathrm{ph}}(T)$ to $\kappa_{\min }(T)$ corresponds to the Ioffe-Regel frequency crossover from the weak phonon scattering regime $\left[\tau_{R}(\omega) \gg \tau_{\min }(\omega)\right]$ to strong phonon scattering $\left[\tau_{R}(\omega) \ll \tau_{\min }(\omega)\right]$. ${ }^{61-66}$ Thus, the IoffeRegel frequency $\omega_{I R}$ can be determined by means of

$$
\tau_{R}\left(\omega_{I R}\right)=\pi \omega_{I R}^{-1}
$$

The term describing the resistive relaxation time $\tau_{R}(\omega, T)$ depends on the solid-state structure and it is clearly different for the OD phase and for the orientationally ordered states. The decomposition of thermal conductivity into two separate contributions has been shown to be adequate to describe this property for monatomic solids ${ }^{52,58}$ and also for molecular solids with rotational degrees of freedom. ${ }^{22,54}$

The framework given by Eq. (2) will be shown to be able to account for the data at hand, and the specifics concerning the diverse mechanisms of heat transport in operation in orientationally disordered and ordered phases are treated in the next sections.

\section{A. Orientationally disordered phases}

The origin of the resistive relaxation time $\tau_{R}(\omega, T)$ for OG is ascribed to resonant phonon scattering and may be described in the frame of the soft potential model. ${ }^{3,68,69}$ The latter (SPM) is cast in terms of a relaxation time $\tau^{-1}(x)$ which can, in turn, be split into three terms using the Matthiesen rule $\tau_{R}^{-1}(\omega)=$ $\tau_{t s}^{-1}+\tau_{c r p}^{-1}+\tau_{l v}^{-1}$ : (i) comprising resonant scattering of sound waves by tunneling states $\left(\tau_{t s}^{-1}\right)$, (ii) classical relaxational processes in asymmetric double-well potentials $\left(\tau_{c r p}^{-1}\right)$, and (iii) localized vibrations (i.e., soft quasiharmonic vibrations) $\left(\tau_{l v}^{-1}\right): 3,19$

$$
\tau_{R}^{-1}(\omega)=\bar{C} \pi \omega \tanh \left(\frac{\hbar \omega}{2 k_{B} T}\right)+\bar{C} \pi \omega\left(\frac{T}{W}\right)^{3 / 4} \ln ^{-1 / 4}\left(\frac{1}{\omega \tau_{0}}\right)+\frac{\bar{C} \pi \omega}{8}\left(\frac{\hbar \omega}{W}\right)^{3},
$$

where $\bar{C}$ is a dimensionless constant accounting for the strength of coupling between a sound wave and the soft localized mode, $\tau_{0}$ is an attempt frequency of the order of $10^{-13} \mathrm{~s}$, and $W$ is the characteristic energy of the quartic term entering the potential of the SPM. This parameter enables the scaling of the elementary excitations in the harmonic soft potential, in such a way that for energies $\hbar \omega \ll W$ localized excitations show up as a two-level system with roughly constant density of states, whereas for $\hbar \omega \geqslant W$ low-energy modes emerge as quasilocal vibrations, dominant at high-temperature, in a soft-single potential. According to Eqs. (3) and (6) the dependence of the thermal conductivity with temperature can be portrayed by means of

$$
\kappa_{\mathrm{ph}}(T)=\frac{2 k_{B} W^{2}}{\pi h \bar{C} c_{s}} F\left(\frac{k_{B} T}{W}\right),
$$

where

$F(z)=\int_{0}^{\infty} d x \frac{x^{3} e^{-x}}{\left(1-e^{-x}\right)^{2}} \frac{z^{2}}{1.1 \tanh (x / 2)+0.7 z^{3 / 4}+x^{3} z^{3} / 8}$,

where $z=k_{B} T / W$ plays the role of a normalized temperature with respect to the SPM potential $(W)$. It is worth mentioning that $F(z)$ provides a universal temperature dependence for the thermal conductivity of glasses, regardless of the disorder frozen in. ${ }^{10}$ The SPM enables the description the behavior of $\kappa_{\mathrm{ph}}(T)$ above $1 \mathrm{~K}$ and up to around $10 \mathrm{~K}$, the temperature domain where a plateau appears, through the assumption of resonant scattering of sound waves from localized lowfrequency vibrations. Below $1 \mathrm{~K}$, it has been shown that the tunneling model $(\mathrm{TM})^{20,69}$ can universally account for the specific heat and thermal conductivity for structural glasses, those devoid of long-range orientational and translational order, in terms of the coexistence of localized tunneling modes with sound waves which gives rise to a constant density of tunneling states. The universality of the thermal conductivity in terms of such a SPM model has been extensively studied for structural glasses ${ }^{3,20,21}$ and also recently for OGs. ${ }^{10}$

The experimental data together with the calculated dependences $\kappa(T), \kappa_{\mathrm{ph}}(T)$ and $\kappa_{\min }(T)$ for the thermal conductivity for the $\mathrm{OG}$ states of $\mathrm{C}_{6} \mathrm{OH}$ and $\mathrm{C}_{6} \mathrm{CN}$ are shown in Fig. 6 . The most relevant result of this figure concerns the remarkable similarity of the thermal conductivity for the OGs of both compounds. The obtained SPM parameters $(W$ and $\bar{C})$ are given in Table II. It is worth mentioning that at high temperatures $\kappa_{\min }(T)$ comes close to the value of heat conductivity for the OD phase I.

Table II compiles the results obtained from model fits to experimental data carried out under the assumption of the SPM through Eq. (6). The similarity between values for the

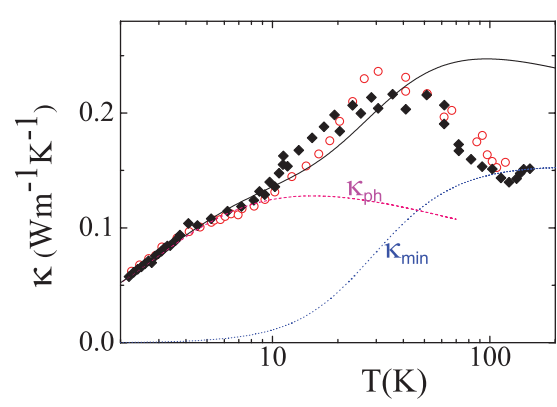

FIG. 6. (Color online) Thermal conductivity as a function of temperature for $\mathrm{OGs} \mathrm{C}_{6} \mathrm{OH}$ (empty circles) and $\mathrm{C}_{6} \mathrm{CN}$ (full diamonds). Dash line (magenta color) is the fitted curve $\kappa_{\mathrm{ph}}(T)$ using the soft-potential model. Dotted line (blue color) is minimal thermal conductivity $\kappa_{\min }(T)$ accordingly to Cahill-Pohl model. Solid line is $\kappa(T)=\kappa_{\mathrm{ph}}(T)+\kappa_{\min }(T)$. 
TABLE II. Parameters of the SPM obtained from the fits of thermal conductivity data for the OGs $\mathrm{C}_{6} \mathrm{OH}$ and $\mathrm{C}_{6} \mathrm{CN}$. Data from literature for the $\mathrm{OG}$ of ethanol $\left(\mathrm{CH}_{3} \mathrm{CH}_{2} \mathrm{OH}\right)$ and freon $112\left(\mathrm{CFCl}_{2}-\mathrm{CFCl}_{2}\right)$ are provided for the sake of comparison. The crossover Ioffe-Regel energy obtained from SPM parameters $\left(\omega_{I R}\right)$ and from the boson peak frequency $\left(\omega_{B P}\right)$ are also given.

\begin{tabular}{|c|c|c|c|c|c|c|c|c|}
\hline Chemical & $\begin{array}{c}\rho \\
\left(\mathrm{kg} \mathrm{m}^{-3}\right)\end{array}$ & $\begin{array}{c}c_{s} \\
\left(\mathrm{~m} \mathrm{~s}^{-1}\right)\end{array}$ & $\begin{array}{l}\Theta_{D} \\
(\mathrm{~K})\end{array}$ & $\begin{array}{c}W / k_{B} \\
(\mathrm{~K})\end{array}$ & $\bar{C} \times 10^{4}$ & $\begin{array}{c}\omega_{I R} \\
(\mathrm{meV})\end{array}$ & $\begin{array}{c}\omega_{B P} \\
(\mathrm{meV})\end{array}$ & Ref. \\
\hline $\mathrm{C}_{6} \mathrm{OH}$ & $967^{\mathrm{a}}$ & $2240^{\mathrm{b}}$ & $\begin{array}{r}120^{\mathrm{c}} \\
115^{\mathrm{d}} \\
95^{\mathrm{g}}\end{array}$ & $\begin{array}{l}3.5 \\
3.0^{\mathrm{e}}\end{array}$ & 4.6 & 3.63 & $\begin{array}{r}\approx 1.4 \\
1.9^{\mathrm{f}} \\
1.8^{\mathrm{e}}\end{array}$ & This work \\
\hline $\mathrm{C}_{6} \mathrm{CN}$ & $1070^{\mathrm{h}}$ & $2100^{b}$ & $\begin{array}{r}113^{\mathrm{h}} \\
93^{\mathrm{d}}\end{array}$ & 3.5 & 4.8 & 3.58 & $\approx 1.4$ & This work \\
\hline Ethanol & & & & 4 & 8.8 & 3.35 & 3.0 & 6 \\
\hline Freon 112 & & & & 2.3 & 2.8 & 2.8 & $\approx 3.5$ & 10 \\
\hline
\end{tabular}

${ }^{\mathrm{a}}$ Reference 28 .

${ }^{\mathrm{b}}$ Calculated from Debye model.

${ }^{\mathrm{c}}$ Reference 25.

${ }^{\mathrm{d}}$ From the effective Debye density of states $g(\omega)=3 \omega^{2} / \omega_{D}^{3}$ (see Fig. 2).

${ }^{e}$ Value estimated from heat capacity of OG (Ref. 31) by means of $\omega_{B P}=5 T_{\max }=21.2 \mathrm{~K}=1.8 \mathrm{meV} ; \mathrm{W}=1.7 T_{\min }=3.0 \pm 0.2 \mathrm{~K}$.

${ }^{\mathrm{f}}$ From Raman spectra; see Fig. 3.

${ }^{g}$ Fitting parameter in Eq. (4) to describe high-temperature plateau on $\kappa(T)$ (see Fig. 6).

${ }^{\mathrm{h}}$ Reference 39.

$W$ parameter for both compounds, which characterizes the crossover from a regime dominated by phonon scattering by low-energy excitations to that governed by soft quasiharmonic vibrations, agrees with the SPM prediction concerning the onset of the plateau at a temperature $T \cong W / k_{B}$. It is also worth mentioning the similarity of the coupling parameter $\bar{C}$ for both compounds, which reveals the virtually identical scattering of low-energy excitations. This result implies that the presence of the $-\mathrm{O}-\mathrm{H}$ and $-\mathrm{C} \equiv \mathrm{N}$ groups does not affect quantitatively such low-energy excitations, which are probably dominated by the librational modes of the molecular ring.

The Ioffe-Regel crossover is observed in thermal conductivity as a deviation of $\kappa(T)$ from $\kappa_{\mathrm{ph}}(T)$ (see Fig. 6). According to the SPM, the Ioffe-Regel crossover, which indicates a transition into a frequency regime where acoustic waves experience strong scattering, is given by $\omega_{I R}=2\left(\pi^{2} \bar{C}\right)^{-1 / 3} W$. Accordingly, values for the Ioffe-Regel crossover are found to be $3.6 \mathrm{meV}$ and $3.5 \mathrm{meV}$ for $\mathrm{C}_{6} \mathrm{OH}$ and $\mathrm{C}_{6} \mathrm{CN}$, respectively.

Fig. 6 shows the temperature region for which the thermal conductivity follows the universal behavior proposed by the SPM, about $k_{B} T / W \leqslant 2$, i.e., for temperatures lower than $\sim 7 \mathrm{~K}$ (see Table II).

\section{B. Orientationally ordered phases}

The resistive relaxation time $\tau_{R}(\omega, T)$ for the ordered phases of $\mathrm{C}_{6} \mathrm{OH}$ and $\mathrm{C}_{6} \mathrm{CN}$ can be accounted for in terms of the action of three mechanisms. These are anharmonic umklapp processes, with relaxation time $\tau_{U}^{-1}$, the scattering by dislocations, $\tau_{\mathrm{dis}}^{-1}$, and the Rayleigh scattering from point lattice defects, $\tau_{\text {imp }}^{-1}$. Thus, assuming the Matthiesen rule, the relaxation time can be written as

$$
\begin{aligned}
\tau_{R}^{-1}(\omega, T) & =\tau_{U}^{-1}+\tau_{\mathrm{dis}}^{-1}+\tau_{\mathrm{imp}}^{-1}, \\
\tau_{U}^{-1} & =B \omega^{2} T \exp \left(\frac{-E_{U}}{T}\right),
\end{aligned}
$$

$$
\begin{aligned}
\tau_{\mathrm{dis}}^{-1}(\omega, T) & =D_{\mathrm{dis}} \omega, \\
\tau_{\mathrm{imp}}^{-1}(\omega, T) & =C_{\mathrm{imp}} \omega^{4},
\end{aligned}
$$

where $B$ represents a frequency factor, $E_{U}$ is the activation energy of the umklapp processes, $D_{\text {dis }}$ is the scattering strength for dislocation, and $C_{\text {imp }}$ is the parameter accounting for the Rayleigh strength. Using Eq. (4) for the whole temperature range, the coefficient $C$ in Eq. (1) should correspond to the high-temperature values of $\kappa(T)$, i.e., with $\kappa_{C P}(T)$ (see Fig. 4). The values of $c_{s}$ and $\theta_{D}$ were obtained from the published specific-heat measurements for $\mathrm{C}_{6} \mathrm{OH}$ and from unpublished specific-heat values of $\mathrm{C}_{6} \mathrm{CN}$ (taken from the Ph.D. dissertation of J. J. Pinvidic ${ }^{39}$ kindly provided by H. Szwarc for the orientationally ordered phase under the assumption of the Debye model; see Table I).

The values for $\kappa_{C P}(T)$ for the orientational ordered states differ from those for OGs by a factor of 0.66 and 1.25 for phases II and III, respectively, for $\mathrm{C}_{6} \mathrm{OH}$ and 2.25 for $\mathrm{C}_{6} \mathrm{CN}$. After the evaluation of the $\kappa_{C P}(T)$ contribution, the $\kappa_{\mathrm{ph}}(T)$ component has been obtained as the difference $\kappa_{\mathrm{ph}}(T)=\kappa(T)-\kappa_{C P}(T)$ and is depicted in Fig. 7.

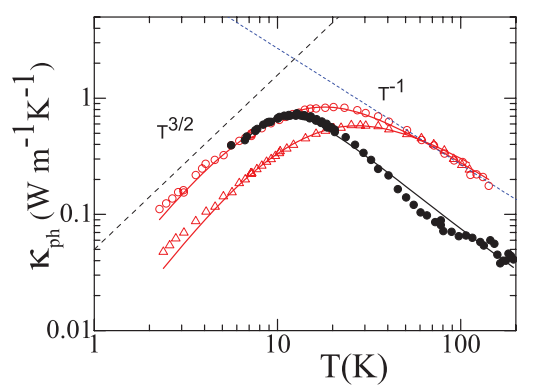

FIG. 7. (Color online) Phonon component $\left[\kappa_{\mathrm{ph}}(T)\right]$ of the thermal conductivity as a function of temperature for orientationally ordered phases of cyclohexanol $\left(\mathrm{C}_{6} \mathrm{OH}\right)$ and cyanocyclohexane $\left(\mathrm{C}_{6} \mathrm{CN}\right)$. Symbols as in Fig. 1. Continuous lines show the fits according to the Debye-Peierls model. 
TABLE III. Fitted values for parameters describing the thermal conductivity of the orientationally ordered phases according to the Debye-Peierls model [see Eqs. (9) and (10)]. Sound velocity $c_{s}$ and Debye temperature $\theta_{D}$ were obtained from specific-heat measurements of Adachi et al. (Ref. 25) and Pinvidic (Ref. 39).

\begin{tabular}{lcccccc}
\hline \hline Chemical & Phase & $\begin{array}{c}c_{s} \\
\left(\mathrm{~m} \mathrm{~s}^{-1}\right)\end{array}$ & $\begin{array}{c}\theta_{D} \\
(\mathrm{~K})\end{array}$ & $\begin{array}{c}B \\
\left(\times 10^{16} \mathrm{~s} \mathrm{~K}^{-1}\right)\end{array}$ & $\begin{array}{c}E_{U} \\
(\mathrm{~K})\end{array}$ & $\begin{array}{c}D_{\mathrm{dis}} \\
\left(\times 10^{3}\right)\end{array}$ \\
\hline $\mathrm{C}_{6} \mathrm{OH}$ & II & 2400 & 136 & 1.7 & 40 & 1.91 \\
& III & 2272 & 133 & 1.5 & 40 & 6.11 \\
$\mathrm{C}_{6} \mathrm{CN}$ & II & 2400 & 136 & 8.5 & 31 & 3.5 \\
\hline \hline
\end{tabular}

The phonon component of the thermal conductivity, $\kappa_{\mathrm{ph}}(T)$, may be quantified using the Debye-Peierls form given by Eq. (3). Inserting Eqs. (10) into Eq. (2) through Eq. (9), the aforementioned parameters were estimated from fits for the $\kappa_{\mathrm{ph}}(T)$ data. Their obtained values are collected in Table III together with the sound velocity $c_{s}$ and Debye temperature $\theta_{D}$. The data shown in Fig. 7 exemplify the ability of the DebyePeierls model to account for the phonon conductivity $\kappa_{\mathrm{ph}}(T)$ of the orientationally ordered phases of $\mathrm{C}_{6} \mathrm{OH}$ and $\mathrm{C}_{6} \mathrm{CN}$. The agreement is especially remarkable at high temperatures, after the phonon maximum, where the $A / T$ law holds.

As regards the physical meaning of such parameters, notice that $B$ characterizes phonon-phonon scattering processes, whereas the parameter $A$ characterizes phonon-phonon scattering process of all sets of phonons, both parameters being connected as $A$ is proportional $B^{-1}$.

The figures quoted above show some striking differences. In particular, the terms describing scattering due to dislocations for the metastable phase III ( $\left.D_{\text {dis }}\right)$ of $\mathrm{C}_{6} \mathrm{OH}$ unveil a larger density of lattice imperfections in its phase III than within phase II. Such a finding correlates with substantial line broadening in the diffraction patterns of phase III which was ascribed to sample strains inherent to the thermodynamic path used to produce such a phase. ${ }^{30}$ Microscopically, the physical mechanism responsible for such a difference can be tentatively ascribed to the number of conformers in the asymmetric unit. The structure of phase III exhibits two molecular conformers, the equatorialin (H-C-O-H dihedral angle of $60^{\circ}$ ) and the equatorial-out ( $\mathrm{H}-\mathrm{C}-\mathrm{O}-\mathrm{H}$ dihedral angle of $180^{\circ}$ ), whereas in phase II only one conformer (equatorial-in) is present in the asymmetric unit. In this respect it is worth pointing out that the increase of the number of conformers within the asymmetric unit in a crystal makes it more difficult to find the thermodynamic path for energy minimization of the system as the temperature is lowered toward the ordered crystal ground state..$^{70,71}$

As far as the ordered phase II of $\mathrm{C}_{6} \mathrm{CN}$ is concerned, the contribution of the umklapp process is much larger than those for the orientationally ordered phases of $\mathrm{C}_{6} \mathrm{OH}$. Because the umklapp process is the anharmonic phonon-phonon scattering process dominant for thermal resistivity providing the $T^{-1}$ "pure" contribution, it can be concluded that this process is responsible for the low values of the thermal conductivity of $\mathrm{C}_{6} \mathrm{CN}$ beyond the phonon maximum if compared to values of the orientationally ordered phases of $\mathrm{C}_{6} \mathrm{OH}$. In addition, delving into the details, the $T^{-1}$ behavior dominates for $T>70 \mathrm{~K}$. The main difference between both materials at a molecular level regards the presence of a bulky $\mathrm{CN}$ group carrying out a strong electric dipole moment. One may guess that, on the grounds of its larger mass than that of the $\mathrm{OH}$ group, the former can more effectively hybridize with the low-lying lattice modes leading to stronger umklapp processes.

It is also worth mentioning that for the orientationally ordered phases II and III of $\mathrm{C}_{6} \mathrm{OH}$ and phase II of $\mathrm{C}_{6} \mathrm{CN}$ $\kappa_{\min }(T)$ values are different, while for the OG states are quite similar. $\kappa_{\min }(T)$ for phase III of $\mathrm{C}_{6} \mathrm{OH}$ is about twice that for phase II. This can be qualitatively explained by the existence of optical phonons contributing to the heat transport. ${ }^{72}$ Under this assumption, $\kappa_{\min }(T)$ can be expressed as

$$
\kappa_{\min }(T)=\kappa_{C P}(T)+\kappa_{o p}(T),
$$

where $\kappa_{o p}(T)$ is the thermal conductivity contribution from optical phonons and strongly depends on the geometrical details of the unit cell of the particular crystal, its temperature dependence being similar to $\kappa_{C P}(T)$.

As regards known differences between the crystal ordered phases let us recall that phase III has a monoclinic cell (space group $P c$ ) with $Z=4$ molecules which pack to form four-membered hydrogen-bonded chains running along the crystallographic $c$ axis. Conversely, phase II shows a tetragonal unit cell (space group $P \overline{4} 2_{1} c$ ) with $Z=8$ molecules in which the molecules form hydrogen-bonded tetramer units. There is one molecule in the asymmetric unit and the hydroxyl groups of the molecules adopt an equatorial conformation. For frequencies relevant for heat transport processes, the optical phonons are expected to involve whole molecule orientational and translational motions within the unit cell and the number of

TABLE IV. The low-frequency Raman and INS spectrum $\left(\mathrm{cm}^{-1}\right)$ of solid cyclohexanol for different temperatures.

\begin{tabular}{lcccccc}
\hline \hline & & & & & Phase III & Phase II \\
& Phase I & OG & OG & & $\begin{array}{c}Z=4 \\
Z=8\end{array}$ \\
& $293 \mathrm{~K}$ & $220 \mathrm{~K}$ & $100 \mathrm{~K}$ & $50 \mathrm{~K}$ & $100 \mathrm{~K}$ & $100 \mathrm{~K}$ \\
\hline 2 & & & & & 106.2 & 107.1 \\
4 & & & & & 93.4 & 84.7 \\
3 & 78.5 & 78.4 & 78.9 & 83.1 & 84.2 & 78.3 \\
5 & 67 & 67.3 & 67.5 & 67.7 & 67.4 & 67.6 \\
7 & & & & 58.9 & & 118.2 \\
1 & & & & & 49.4 & 49.8 \\
6 & & & & & 73.3 & 25.5 \\
10 & & & & & & 42.2 \\
11 & & & & & 34.5 \\
8 & & & & & 74.6 \\
9 & & & & & & \\
\hline \hline
\end{tabular}




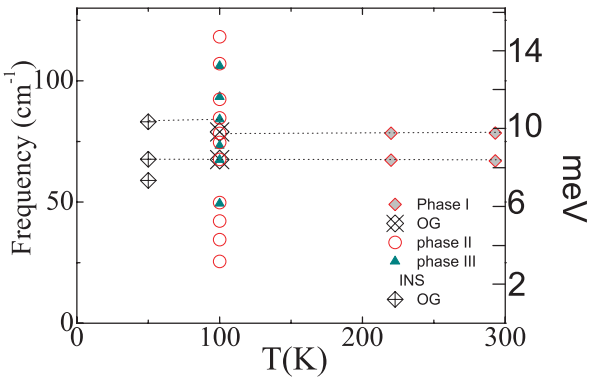

FIG. 8. (Color online) Temperature dependence of the lowfrequency Raman and INS spectrum of solid cyclohexanol.

the local frequencies is given by $3(2 z-1)$. The low-frequency Raman spectrum (see Fig. 5) only shows the subset of Raman-active modes from the spectrum of optical phonons. The measured Raman frequencies for $\mathrm{C}_{6} \mathrm{OH}$ are given in Table IV and then shown in Fig. 8 as a correlation chart. Our thermal conductivity results show that low-frequency optical modes in phase II and phase III will influence the value of $\kappa_{\mathrm{ph}}(T)$ although they seem to be more important to explain the achieved values of $\kappa_{\min }(T)$.

Before the structural study by Ibberson et $a l^{30}$ was available, the larger $\kappa(T)$ in phase III than in phase II was alternatively explained by Andersson et al. ${ }^{32}$ under the assumption that phases II and III are not well-ordered as a result of a mixture of several conformations for the $\mathrm{C}_{6} \mathrm{OH}$ molecule.

\section{CONCLUSIONS}

The striking differences concerning the extent in temperature of the thermal conductivity plateau for samples under study are here ascribed to differences in the potential energy landscapes associated with molecular degrees of freedom which hybridize with sound modes, and thus provide extra channels for scattering of long-wavelength phonons. On these grounds, the large plateau found for several molecular SGs or OGs is explained as a result of a combination of relaxational scattering from quasilocal vibrations and phonon scattering originating from librational motions emerging at the tail of the density of states. ${ }^{5,7,10}$ As for the orientationally disordered phases of $\mathrm{C}_{6} \mathrm{OH}$ and $\mathrm{C}_{6} \mathrm{CN}$, the plateau extension is limited to a narrow temperature domain, and thus the potential energy landscape for both compounds should be very close, evidence which agrees with the similarity of the molecular degrees of freedom.

Finally, we have also shown that orientational glasses behave similarly to canonical glass formers as far as the Ioffe-Regel limit is concerned. The crossover Ioffe-Regel energy limit obtained from the conductivity data by application of the soft-potential model does not correlate with the boson peak energy obtained from inelastic neutron scattering through the maximum of the $g(\omega) / \omega^{2}$ function. As for cyclohexanol and cyanocyclohexane orientational glasses, the obtained Ioffe-Regel energy crossover $\left(\omega_{I R}\right)$ is higher than that of the boson peak energy $\left(\omega_{B P}\right)$, in agreement with the recent results reported by Ruocco et al. ${ }^{67}$

\section{ACKNOWLEDGMENTS}

We acknowledge H. Szwarc for kindly provide us the Ph.D. dissertation of J. J. Pinvidic containing the unpublished specific-heat data for cyanocyclohexane. We thank T. Unruh for technical advice in neutron measurements at the TOFTOF line at FRM II (Garching, Germany). This work was financially supported in part by the Spanish Ministry of Science and Innovation (Grant No. FIS2008-00837) and the Catalan Government (Grant No. 2009SGR-1251). *jose.luis.tamarit@upc.edu

${ }^{1}$ R. O. Pohl, X. Liu, and E. Thompson, Rev. Mod. Phys. 74, 991 (2002).

${ }^{2}$ F. Bridges and L. Downward, Phys. Rev. B 70, 140201R (2004).

${ }^{3}$ U. Buchenau, Yu. M. Galperin, V. L. Gurevich, D. A. Parshin, M. A. Ramos, and H. R. Schober, Phys. Rev. B 46, 2798 (1992).

${ }^{4}$ J. L. Feldman, P. B. Allen, and S. R. Bickham, Phys. Rev. B 59, 3551 (1999).

${ }^{5}$ A. I. Krivchikov, A. N. Yushchenko, O. A. Korolyuk, F. J. Bermejo, C. Cabrillo, and M. A. González, Phys. Rev. B 75, 214204 (2007).

${ }^{6}$ A. I. Krivchikov, A. N. Yushchenko, V. G. Manzhelii, O. A. Korolyuk, F. J. Bermejo, R. Fernandez-Perea, C. Cabrillo, and M. A. González, Phys. Rev. B 74, 060201R (2006).

${ }^{7}$ A. I. Krivchikov, A. N. Yushchenko, O. A. Korolyuk, F. J. Bermejo, R. Fernandez-Perea, I. Bustinduy, and M. A. González, Phys. Rev. B 77, 024202 (2008).

${ }^{8}$ R. Brand, P. Lunkenheimer, and A. Loidl, J. Chem. Phys. 116, 10386 (2002).

${ }^{9}$ A. Criado, M. Jiménez-Ruiz, C. Cabrillo, F. J. Bermejo, R. Fernández-Perea, H. E. Fischer, and F. R. Trouw, Phys. Rev. B 61, 12082 (2000).
${ }^{10}$ I. V. Sharapova, A. I. Krivchikov, O. A. Korolyuk, A. Jezowski, M. Rovira-Esteva, J. L1. Tamarit, L. C. Pardo, M. D. Ruiz-Martin, and F. J. Bermejo, Phys. Rev. B 81, 094205 (2010).

${ }^{11}$ A. Drozd-Rzoska, S. J. Rzoska, S. Pawlus, and J. Ll. Tamarit, Phys. Rev. B 73, 224205 (2006).

${ }^{12}$ M. D. Ediger, C. A. Angell, and S. R. Nagel, J. Phys. Chem. 100, 13200 (1996).

${ }^{13}$ A. I. Chumakov, G. Monaco, A. Monaco, W. A. Crichton, A. Bosak, R. Rüffer, A. Meyer, F. Kargl, L. Comez, D. Fioretto, H. Giefers, S. Roitsch, G. Wortmann, M. H. Manghnani, A. Hushur, Q. Williams, J. Balogh, K. Parlinski, P. Jochym, and P. Piekarz, Phys. Rev. Lett. 106, 225501 (2011).

${ }^{14}$ T. Grigera, V. Martin-Mayor, G. Parisi, and P. Verrocchio, Nature (London) 422, 289 (2003).

${ }^{15}$ W. Schirmacher, G. Diezemann, and C. Ganter, Phys. Rev. Lett. 81, 136 (1998).

${ }^{16}$ W. Schirmacher, G. Ruocco, and T. Scopigno, Phys. Rev. Lett. 98, 025501 (2007)

${ }^{17}$ S. N. Taraskin, Y. L. Loh, G. Natarajan, and S. R. Elliott, Phys. Rev. Lett. 86, 1255 (2001).

${ }^{18}$ V. L. Gurevich, D. A. Parshin, and H. R. Schober, Phys. Rev. B 67, 094203 (2003). 
${ }^{19}$ M. A. Ramos and U. Buchenau, Phys. Rev. B 55, 5749 (1997).

${ }^{20}$ U. Buchenau, J. Phys. Condens. Matter 13, 7827 (2001).

${ }^{21}$ V. G. Karpov, M. I. Klinger, and F. N. Ignatiev, Solid State Commun. 44, 333 (1982).

${ }^{22}$ A. I. Krivchikov, I. V. Sharapova, O. A. Korolyuk, O. O. Romantsova, and F. J. Bermejo, Low Temp. Phys. 35, 0891 (2009).

${ }^{23}$ D. A. Parshin, Phys. Rev. B 49, 9400 (1994).

${ }^{24}$ R. O. Pohl, Phys. Rev. Lett. 8, 481 (1962).

${ }^{25}$ K. Adachi, H. Suga, and S. Seki, Bull. Chem. Soc. Jpn. 41, 1073 (1968); T. Matsuo, H. Suga, and S. Seki, ibid. 39,1837 (1966); K. Kishimoto, H. Suga, and S. Seki, ibid. 53, 2748 (1980); K. K. Kelley, J. Am. Chem. Soc. 51, 1400 (1929); A. Dworkin, A. H. Fuchs, M. Ghelfenstein, and H. Szwarc, J. Phys. (France) 43, L21 (1982); K. Adachi, H. Suga, S. Seki, S. Kubota, S. Yamaguchi, O. Yano, and Y. Wada, Mol. Cryst. Liq. Cryst. 18, 345 (1972); M. Mizukami, H. Fujimori, and M. Oguni, Solid State Comm. 100, 83 (1996).

${ }^{26}$ M. Shablakh, L. A. Dissado, and R. M. Hill, J. Chem. Soc., Faraday Trans. 2 79, 369 (1983); Gangasharan and S. S. N. Murthy, J. Chem. Phys. 99, 9865 (1993); T. Shinomiya, Bull. Chem. Soc. Jpn. 63, 1087 (1990).

${ }^{27}$ P. L. Kuhns and M. S. Conradi, J. Chem. Phys. 80, 5851 (1984).

${ }^{28}$ D. André, D. Ceccaldi, and H. Szwarc, J. Phys. (France) 45, 731 (1984).

${ }^{29}$ D. Ceccaldi, Phys. Rev. B 31, 8221 (1985).

${ }^{30}$ R. M. Ibberson, S. Parsons, D. R. Allan, and A. M. T. Bell, Acta Cryst. B 64, 573 (2008).

${ }^{31}$ E. Bonjour, R. Calemczuk, R. Lagnier, and B. Salce, J. Phys. Coll. 42, C6-63 (1981).

${ }^{32}$ O. Andersson, R. G. Ross, and G. Backstrom, Mol. Phys. 66, 619 (1989).

${ }^{33}$ H.-J. Scheneider and V. Hoppen, J. Org. Chem. 43, 3866 (1978); Ch. Jansen, D. an Mey, G. Raabe, and J. Fleischhauer, J. Mol. Struct. 398-399, 2395 (1997); E. Kleinpeter and F. Taddei, ibid. 585, 223 (2002); U. John and K. P. R. Nair, Spectrochim. Acta A 61, 2555 (2005).

${ }^{34}$ A. Gonthier-Vassal and H. Szwarc, Chem. Phys. Lett. 129, 5 (1986).

${ }^{35}$ J. Reuter, T. Brückert, and A. Würflinger, Z. Naturforsch. Teil A 48, 705 (1993).

${ }^{36} \mathrm{C}$. Tschirwitz, S. Benkhof, T. Blochowicz, and E. Rössler, J. Chem. Phys. 117, 6281 (2002).

${ }^{37}$ L. P. Singh and S. S. N. Murthy, J. Chem. Phys. 129, 094501 (2008).

${ }^{38}$ N. V. Surovtsev, S. V. Adichtchev, J. Wiedersich, V. N. Novikov, and E. A. Rossler, J. Chem. Phys. 119, 12399 (2003).

${ }^{39}$ J. J. Pinvidic, Ph.D. thesis, Université de Paris Sud, 1988.

${ }^{40}$ K. Kishimoto, J. J. Pinvidic, T. Matsuo, and H. Suga, Proc. Jpn. Acad., Ser. B: Phys. Biol. Sci. 67, 66 (1991); H. Suga, J. Mol. Liq. 65/66, 115 (1995); Thermochim. Acta 245, 69 (1994).

${ }^{41}$ J. R. Durig, R. M. Ward, A. R. Conrad, M. J. Tubergen, K. G. Nelson, P. Groner, and T. K. Gounev, J. Mol. Struct. 967, 99 (2010).

${ }^{42}$ T. Woldbaek, A. Berkessel, A. Horn, and P. Klaeboe, Acta Chem. Scand. A36, 719 (1982).

${ }^{43}$ H. T. Horntvedt and P. Klaeboe, Acta Chem. Scand. A29, 528 (1975).
${ }^{44}$ A. I. Krivchikov, V. G. Manzhelii, O. A. Korolyuk, B. Ya. Gorodilov, and O. O. Romantsova, Phys. Chem. Chem. Phys. 7, 728 (2005).

${ }^{45}$ V. G. Manzhelii and Y. A. Freeman, The Physics of Cryocrystals (AIP, Woodbury, NY, 1997), p. 500.

${ }^{46}$ IDA program, [http://sourceforge.net/projects/frida/]. Tutorial can be found at [http://gcm.upc.edu/members/luis-carlos/frida1].

${ }^{47}$ V. Z. Gochiyaev, V. K. Malinovsky, V. N. Novikov, and A. P. Sokolov, Philos. Mag. B 63, 777 (1991).

${ }^{48} \mathrm{R}$. Berman, Thermal Conduction in Solids (Clarendon Press, Oxford 1976).

${ }^{49}$ R. G. Ross, P. Andersson, B. Sundqvist, and G. Backstrom, Rep. Prog. Phys. 47, 1347 (1984).

${ }^{50}$ A. F. Chudnovskii, B. M. Mogilevskii, and V. G. Surin, J. Eng. Phys. Thermophys. 19, 1295 (1970).

${ }^{51}$ O. A. Korolyuk, Low Temp. Phys. 37, 416 (2011).

${ }^{52}$ J. Callaway, Phys. Rev. 113, 1046 (1959).

${ }^{53}$ V. A. Slusarev and Yu A. Freiman, J. Low. Temp. Phys. 9, 657 (1972).

${ }^{54}$ V. A. Konstantinov, Low Temp. Phys. 29, 422 (2003).

${ }^{55}$ V. A. Konstantinov, V. G. Manzhelii, M. A. Strzhemechny, and S. A. Smirnov, Fiz. Nizk. Temp. 14, 90 (1988).

${ }^{56}$ A. Jagannathan, R. Orbach, and O. Entin-Wohlman, Phys. Rev. B 39, 13465 (1989).

${ }^{57}$ P. B. Allen and J. L. Feldman, Phys. Rev. B 48, 12581 (1993).

${ }^{58}$ A. J. H. McGaughey and M. Kaviany, Phys. Rev. B 69, 094303 (2004).

${ }^{59}$ T. Sun and P. B. Allen, Phys. Rev. B 82, 224305 (2010).

${ }^{60}$ D. G. Cahill and R. O. Pohl, Annu. Rev. Phys. Chem. 39, 93 (1988); Phys. Rev. B 35, 4067 (1987); D. G. Cahill, S. K. Watson, and R. O. Pohl, ibid. 46, 6131 (1992).

${ }^{61}$ J. J. Freeman and A. C. Anderson, Phys. Rev. B 34, 5684 (1986).

${ }^{62}$ M. I. Klinger and V. Halpern, Phys. Lett. A 313, 448 (2003); M. I. Klinger and A. M. Kosevich, ibid. 280, 365 (2001); 295, 311 (2002).

${ }^{63}$ A. F. Ioffe and A. R. Regel, Prog. Semicond. 4, 237 (1960).

${ }^{64}$ S. R. Elliot, Europhys. Lett. 19, 201 (1992).

${ }^{65}$ P. B. Allen, J. L. Feldman, J. Fabian, and F. Wooten, Philos. Mag. B 72, 1715 (1999).

${ }^{66}$ B. Rufflé, G. Guimbretière, E. Courtens, R. Vacher, and G. Monaco, Phys. Rev. Lett. 96, 045502 (2006).

${ }^{67}$ G. Ruocco, A. Matic, T. Scopigno, and S. N. Yannopoulos, Phys. Rev. Lett. 98, 079601 (2007).

${ }^{68}$ U. Buchenau, Yu. M. Galperin, V. L. Gurevich, and H. R. Schober, Phys. Rev. B 43, 5039 (1991).

${ }^{69}$ L. Gil, M. A. Ramos, A. Bringer, and U. Buchenau, Phys. Rev. Lett. 70, 182 (1993).

${ }^{70}$ M. Bujakm, D. Bläser, A. Katrusiak, and R. Boese, Chem. Comm. 47, 8769 (2011).

${ }^{71}$ M. A. Rute, J. Salud, D. O. Lopez, J. Ll. Tamarit, Ph. Negrier, M. Barrio, and D. Mondieig, Chem. Mater. 15, 4725 (2003).

${ }^{72}$ A. J. H. McGaughey and M. Kaviany, Int. J. Heat Mass Transfer 47, 1799 (2004); B. L. Huang and M. Kaviany, 77, 125209 (2008). 